\title{
INTERPRETATION OF THE ARCADE 2 ABSOLUTE SKY BRIGHTNESS MEASUREMENT
}

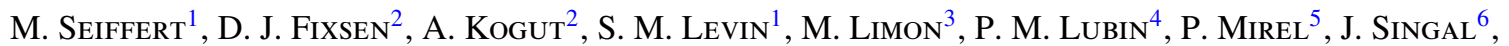 \\ T. Villela ${ }^{7}$, E. Wollack ${ }^{2}$, And C. A. Wuensche ${ }^{7}$ \\ ${ }^{1}$ Jet Propulsion Laboratory, 4800 Oak Grove Drive, Pasadena, CA 91109, USA; Michael.D.Seiffert@jpl.nasa.gov \\ ${ }^{2}$ University of Maryland, Code 665, Goddard Space Flight Center, Greenbelt, MD 20771, USA \\ ${ }^{3}$ Columbia Astrophysics Laboratory, 550W 120th St., Mail Code 5247, New York, NY 10027-6902, USA \\ ${ }^{4}$ Physics Department, University of California, Santa Barbara, CA, USA \\ ${ }^{5}$ Wyle Informations Systems, Code 665, Goddard Space Flight Center, Greenbelt, MD 20771, USA \\ ${ }^{6}$ Kavli Institute for Particle Astrophysics and Cosmology, SLAC National Accelerator Laboratory, Menlo Park, CA 94025, USA \\ ${ }^{7}$ Instituto Nacional de Pesquisas Espaciais, Divisão de Astrofísica, Caixa Postal 515, 12245-970-São José dos Campos, SP, Brazil \\ Received 2011 February 28; accepted 2011 March 28; published 2011 May 17
}

\begin{abstract}
We use absolutely calibrated data between 3 and $90 \mathrm{GHz}$ from the 2006 balloon flight of the ARCADE 2 instrument, along with previous measurements at other frequencies, to constrain models of extragalactic emission. Such emission is a combination of the cosmic microwave background (CMB) monopole, Galactic foreground emission, the integrated contribution of radio emission from external galaxies, any spectral distortions present in the CMB, and any other extragalactic source. After removal of estimates of foreground emission from our own Galaxy, and an estimated contribution of external galaxies, we present fits to a combination of the flat-spectrum CMB and potential spectral distortions in the CMB. We find $2 \sigma$ upper limits to CMB spectral distortions of $\mu<6 \times 10^{-4}$ and $\left|Y_{\mathrm{ff}}\right|<1 \times 10^{-4}$. We also find a significant detection of a residual signal beyond that, which can be explained by the CMB plus the integrated radio emission from galaxies estimated from existing surveys. This residual signal may be due to an underestimated galactic foreground contribution, an unaccounted for contribution of a background of radio sources, or some combination of both. The residual signal is consistent with emission in the form of a power law with amplitude $18.4 \pm 2.1 \mathrm{~K}$ at $0.31 \mathrm{GHz}$ and a spectral index of $-2.57 \pm 0.05$.
\end{abstract}

Key words: cosmic background radiation - cosmology: observations

\section{INTRODUCTION}

The cosmic microwave background (CMB) is currently our most precise window on the physics of the early universe. Measurements of the frequency spectrum of the CMB can rule out alternative cosmologies and place limits on physical processes that may distort the spectrum, including dark matter particle decay and reionization. Departures from a thermal blackbody spectrum are expected at a small level from a variety of mechanisms.

The Cosmic Background Explorer satellite observed the spectrum of the CMB with the Far-Infrared Absolute Spectrophotometer (FIRAS) instrument (Mather et al. 1990) at wavelengths between $1 \mathrm{~cm}$ and $100 \mu \mathrm{m}$. FIRAS results reported by Fixsen et al. (1996), Mather et al. (1999), and Fixsen \& Mather (2002) are consistent with a blackbody spectrum at a temperature of $T_{\mathrm{CMB}}=2.725 \pm 0.001 \mathrm{~K}$.

Absolutely calibrated measurements of the CMB at longer wavelengths (lower frequency) than FIRAS have been performed with ground-based and balloon-borne experiments. Among the most sensitive of these measurements are those of Johnson \& Wilkinson (1987), Levin et al. (1992), Bersanelli et al. (1994, 1995), Staggs et al. (1996a, 1996b), Raghunathan \& Subrahmnayan (2000), Fixsen et al. (2004), Singal et al. (2006), and Zannoni et al. (2008).

The second generation of the Absolute Radiometer for Cosmology, Astrophysics, and Diffuse Emission (ARCADE 2) was conceived as a balloon-borne experiment to improve constraints on spectral distortions in the $\mathrm{CMB}$, with particular emphasis on the $3-10 \mathrm{GHz}$ frequency range. ARCADE 2 uses a unique, clear aperture instrument design with the bulk of the instrument operating at or near the temperature of the CMB. This minimizes the potential contribution to instrument systematics from warm, emissive optics. The instrument uses a set of microwave feed horns to compare the sky to a large, cryogenic blackbody calibration target. The results described here are from the second version of the instrument, described in detail by Singal et al. (2011). The sky measurements from the second flight of this instrument are described by Fixsen et al. (2011), in which the authors present a detection of the sky temperature consistent with a blackbody CMB plus an isotropic power-law residual. The model of Galactic emission used in interpreting the ARCADE 2 data is described by Kogut et al. (2011).

In this paper, we use the combination of ARCADE 2 and other data sets to present a detection of $3 \mathrm{GHz}$ emission in excess of that expected from the $\mathrm{CMB}$, galactic foreground emission, and existing source counts of radio galaxies. We correct the results of Fixsen et al. (2011) to remove the modeled radio background from known populations of discrete radio galaxies and fit the remaining isotropic emission to a combination of CMB and radio emission. The resulting amplitude and spectral index for the radio excess thus differ from Fixsen et al. (2011), which include the contribution from radio galaxies. We also use the combined data to place limits on spectral distortions to the $\mathrm{CMB}$ and show that canonical spectral distortions cannot explain the excess emission.

This paper is organized as follows. Section 2 summarizes estimates of isotropic, residual emission at a variety of frequencies that we have used in our analysis. Section 3 examines the potential contribution of extragalactic point sources and their potential to affect our conclusions. Section 4 presents our spectral fits to the data and our limits on spectral distortions of the 
Table 1

Measurements of Radio Emission

\begin{tabular}{lcccc}
\hline \hline Frequency $(\mathrm{GHz})$ & $\begin{array}{c}\text { Extragalactic }^{\mathrm{a}} \\
\text { Temperature }(\mathrm{K})\end{array}$ & Error $^{\mathrm{b}}(\mathrm{K})$ & $\begin{array}{c}\text { Residual }^{\mathrm{c}} \\
\text { Emission }(\mathrm{K})\end{array}$ & Error $^{\mathrm{b}}$ \\
\hline 0.022 & 20355 & 5181 & 13268 & 5229 \\
0.045 & 3864 & 502 & 2843 & 512 \\
0.408 & 13.42 & 3.52 & 10.80 & 3.53 \\
1.42 & 3.271 & 0.526 & 3.181 & 0.526 \\
3.20 & 2.787 & 0.010 & 2.777 & 0.010 \\
3.41 & 2.770 & 0.008 & 2.761 & 0.008 \\
7.98 & 2.761 & 0.013 & 2.761 & 0.013 \\
8.33 & 2.743 & 0.015 & 2.742 & 0.015 \\
9.72 & 2.731 & 0.005 & 2.730 & 0.005 \\
10.49 & 2.738 & 0.006 & 2.738 & 0.006 \\
29.5 & 2.529 & 0.155 & 2.529 & 0.155 \\
31 & 2.573 & 0.076 & 2.573 & 0.076 \\
90 & 2.706 & 0.019 & 2.706 & 0.019 \\
250 & 2.725 & 0.001 & 2.725 & 0.001 \\
\hline
\end{tabular}

Notes.

a This is our estimate of the monopole temperature with the Milky Way Galactic contribution removed as by Kogut et al. (2011). Data are from Roger et al. (1999; $0.022 \mathrm{GHz}$ ), Maeda et al. (1999; $0.045 \mathrm{GHz})$, Haslam et al. (1981; $0.408 \mathrm{GHz}$ ), Reich \& Reich (1986; $1.42 \mathrm{GHz}$ ), and Fixsen et al. (2011; 3.20 to $90 \mathrm{GHz})$. The FIRAS data set has been condensed to a single data point at $250 \mathrm{GHz}$ (see the text). All temperature values in this table are quoted as thermodynamic temperatures.

$\mathrm{b}$ There is a correlation among the errors due to the nature of the Galaxy model. Errors reported in this table represent the square root of diagonal elements of the covariance matrix (Table 5 in Fixsen et al. 2011) only. The spectral fits described in the text use the full covariance.

${ }^{c}$ Residual isotropic emission after subtraction of an estimate of the contribution of extragalactic discrete radio sources from Gervasi et al. (2008a) model "Fit1." We have adopted a $10 \%$ fractional error for this contribution.

CMB. Section 5 presents discussion of the results, including potential explanations for the source of the excess emission.

\section{RESULTS FROM ARCADE 2 AND OTHER SURVEYS}

For our analysis, we use the data from the 2006 flight of the ARCADE 2 instrument, from FIRAS, and from lower frequency ground-based surveys. FIRAS measures a highprecision difference between the sky and a calibrated reference target. The result is a set of values with tiny relative errors, and a larger, $1 \mathrm{mK}$ calibration error common to all the data points. Table 1 summarizes the data used in our analysis, which includes ARCADE 2, the $22 \mathrm{MHz}$ survey of Roger et al. (1999), the $45 \mathrm{MHz}$ survey of Maeda et al. (1999), the $408 \mathrm{MHz}$ survey of Haslam et al. (1981), and the $1.42 \mathrm{GHz}$ survey of Reich \& Reich (1986). We remove a model of Galactic emission from these data sets as described in Kogut et al. (2011). The data in Table 1 are the resulting estimate of the residual, isotropic emission. The ARCADE 2 data in the $3-10 \mathrm{GHz}$ range are shown in Figure 1; they lie significantly above the $2.725 \mathrm{~K}$ blackbody CMB determined by FIRAS at higher frequencies.

In our analysis, we have excluded the $100-200 \mathrm{MHz}$ results of Rogers \& Bowman (2008). They find a minimum diffuse background of $237 \mathrm{~K}$ at $150 \mathrm{MHz}$, but their work does not provide an independent estimate of the Galactic contribution. We can, however, check for consistency by using the Galactic model described by Kogut et al. (2011) extrapolated to $150 \mathrm{MHz}$, where we find an approximately $60 \mathrm{~K}$ Galactic contribution to the diffuse background in the region of high Galactic latitude. If we subtract this Galactic contribution from the $150 \mathrm{MHz}$ data,

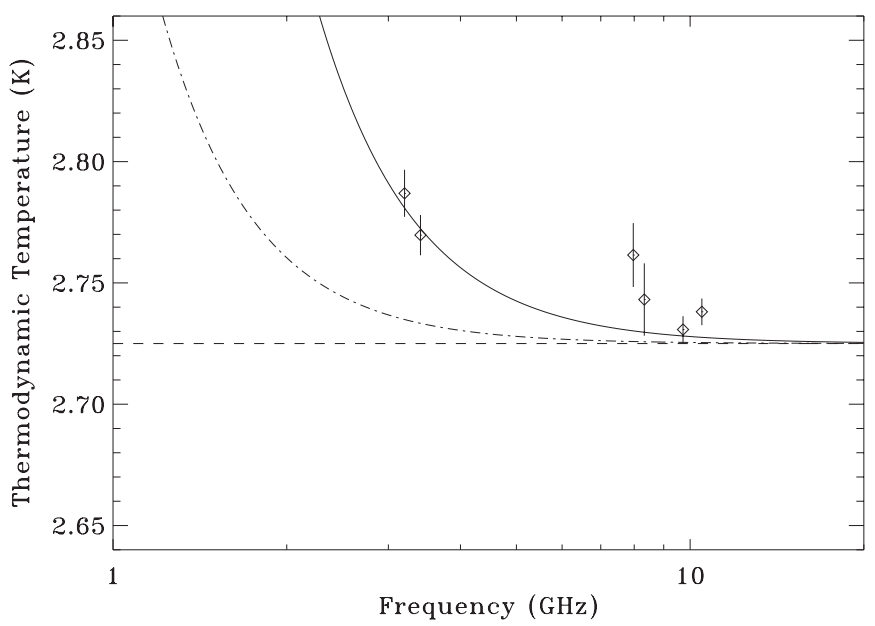

Figure 1. Detection of radio emission by ARCADE 2 beyond the contribution of discrete radio sources, modeled galactic foregrounds, and the expectation of $2.725 \mathrm{~K}$ blackbody radiation. Data points are the ARCADE 2 results from Fixsen et al. (2011) and have been corrected for Milky Way Galactic emission described by Kogut et al. (2011). The dashed curve is a constant $2.725 \mathrm{~K}$ blackbody, consistent with FIRAS measurements of the CMB. The dot-dashed curve is an estimate of the discrete radio source contribution from Gervasi et al. (2008a) model "Fit1" added to the 2.725 blackbody. The data points lie significantly above this dot-dashed curve, indicating our detection of excess emission. The solid curve is the best fit of the combined data of Table 1 and FIRAS to a power law plus a constant $\mathrm{CMB}$ temperature.

the resulting amplitude is consistent with the model fit presented in Section 4.

Our results are also consistent with the measurement reported by Staggs et al. (1996b), provided we adopt the Galactic modeling described by Kogut et al. (2011), rather than their original extrapolation of $408 \mathrm{MHz}$ data. The difference between these two models is essentially a question of where to draw the line for Galactic versus extragalactic emission. Staggs et al. (1996b) model Galactic emission by taking the $408 \mathrm{MHz}$ survey, convolving it with their beam pattern, subtracting $2.74 \mathrm{~K}$ for the $\mathrm{CMB}$, and scaling the remaining signal with a spectral index -2.8 . They explicitly note that this lumps together any Galactic and extragalactic signals assuming that the extragalactic radio background has a similar spectral index. Therefore, their "galactic" model is equivalent to the sum of our Galactic model plus the isotropic residual. By contrast, we explicitly separate the $408 \mathrm{MHz}$ survey data (along with the other surveys and the ARCADE data) into separate Galactic and extragalactic pieces.

We have not included a number of other measurements, including the rocket-borne measurements of Gush et al. (1990) and the ground-based and balloon-borne measurements cited earlier. The size of the uncertainties quoted in these measurements results in no significant contribution to the constraints on our model fits.

\section{CONTRIBUTION OF SOURCES}

The set of measurements in Table 1 does not have sufficient angular resolution to reject discrete radio sources. Instead, we must estimate the contribution of these sources through one of the two ways: direct radio surveys designed to detect such sources or measurements of the far-IR background which can trace the integrated emission of such sources through the correlation of the far-IR and radio emission. We examine these two methods in turn. 


\subsection{Expectation from Source Counts}

The sky brightness temperature contributed by discrete sources can be composed as the sum of two parts: the source population that has been characterized by existing surveys and the contribution of sources below the flux limit of existing surveys. We write this as

$$
T=T\left(S>S_{\text {limit }}\right)+\frac{\lambda^{2}}{2 k_{\mathrm{B}}} \int_{S_{\min }}^{S_{\text {limit }}} \frac{d N}{d S} S d S,
$$

where $T\left(S>S_{\text {limit }}\right)$ is contribution from sources with a flux $S$ greater than the survey limit $S_{\text {limit. }}$. The wavelength of observation is $\lambda$ and $k_{\mathrm{B}}$ is the Boltzmann constant. We characterize sources below the survey limit with their differential number counts, $d N / d S$, and assume that there is a lower limit cutoff to the source population at a flux of $S_{\min }$. Radio source count surveys reveal a faint-source population with differential number counts proportional to a power law

$$
\frac{d N}{d S}=\kappa S^{-\gamma},
$$

where $\kappa$ is the constant of proportionality and $\gamma$ is the powerlaw index. An index $\gamma$ of 2.5 corresponds to a static, Euclidean universe with uniform filling of sources, whereas faint radio surveys find $\gamma$ in the range of 2.0-2.6 (Windhorst et al. 1993; Richards 2000; Fomalont et al. 2002, 2006; Kellerman et al. 2008; Owen \& Morrison 2008).

Such source counts cannot extend to arbitrarily low fluxes, or the total contribution would diverge. A realistic distribution of sources, of course, would not have a sharp cutoff at $S_{\min }$. In practice, we can characterize $S_{\min }$ as the flux below which the index $\gamma$ falls below 2, as there will be negligible additional contribution to the integral below this limit.

Deep surveys of radio sources have been performed at a number of frequencies. Particularly useful are the surveys at 1.4 and $8.4 \mathrm{GHz}$ with the Very Large Array (VLA). Fomalont et al. (2002) report the results of an $8.4 \mathrm{GHz}$ survey, which we will examine as an example. This survey, with a limit of $7.5 \mu \mathrm{Jy}$, finds a faint-end index to the number counts of $\gamma=2.11 \pm 0.13$. Windhorst et al. (1993) argue that the sources in the nJy flux range are dominated by ordinary spiral galaxies, which produces a natural lower limit of $30 \mathrm{nJy}$; below this limit there are insufficient galaxies.

Figure 2 shows a range of estimates for the contribution of discrete sources to the ARCADE $23.20 \mathrm{GHz}$ measurement. We have calculated the expected contribution to the extragalactic sky temperature using the results of Fomalont et al. (2002) and varying the faint-end index, and plotting as a function of $S_{\min }$. We have scaled the temperature from 8.4 to $3.2 \mathrm{GHz}$ using a frequency spectral index of -2.75 . This spectral index is characteristic of starburst and normal spiral galaxies with synchrotron emission, though at $8.4 \mathrm{GHz}$ there could be some contribution from flat-spectrum sources such as active galactic nuclei. The sources fainter than $35 \mu \mathrm{Jy}$ described by Fomalont et al. (2002) have a spectral index distribution that peaks at -2.75 . From this analysis, we conclude that the contribution is likely in the range of 5-10 $\mathrm{mK}$ at $3.20 \mathrm{GHz}$, which should be compared to the $62 \pm 10 \mathrm{mK}$ excess measured by ARCADE 2 . We also note that the normalization of the differential number counts in the $8.4 \mathrm{GHz}$ survey is sufficiently accurate to not contribute a significant source of error to this analysis.

We have focused on the $8.4 \mathrm{GHz}$ survey results as illustrative; these measurements are amenable to extrapolation to fainter

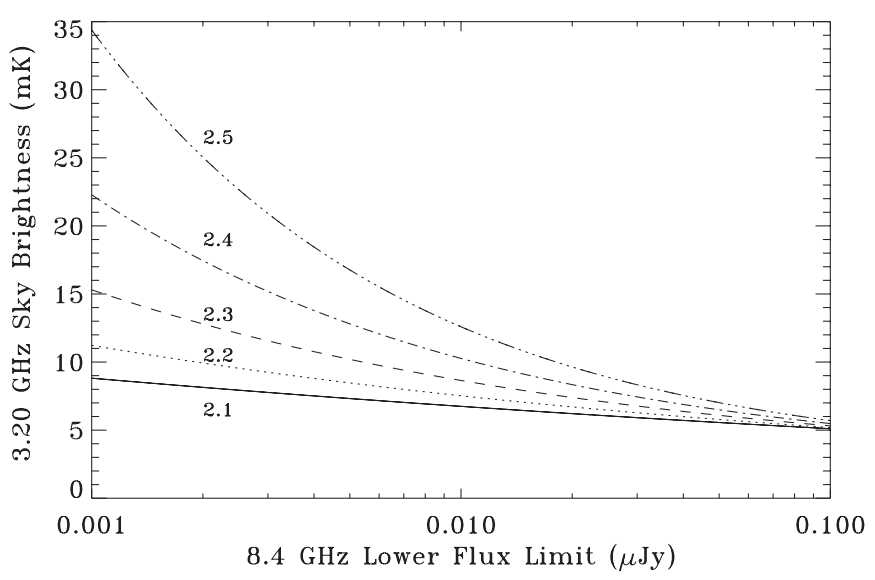

Figure 2. Estimated contribution of extragalactic source counts to the sky brightness at $3.20 \mathrm{GHz}$ vs. the assumed faint-end cutoff of $8.4 \mathrm{GHz}$ source counts. The sky brightness is first calculated at $8.4 \mathrm{GHz}$ from the sum of the existing source population greater than $7.5 \mu \mathrm{Jy}$ (Fomalont et al. 2002) and the contribution of fainter sources assuming a continuation of the differential number counts with a power-law index of 2.1 (solid curve), 2.2 (dotted curve), 2.3 (dashed curve), 2.4 (dash dot curve), and 2.5 (dash-dot-dot curve). The contribution is scaled to the ARCADE $3.20 \mathrm{GHz}$ channel with a frequency index of -2.75 , as is typical of sources in faint radio surveys. The measured index of differential number counts at the faint end of the $8.4 \mathrm{GHz}$ survey is $\gamma=2.11 \pm 0.13$. The total contribution indicated in the plot should be compared to the $62 \pm 10 \mathrm{mK}$ excess signal measured by ARCADE 2 at $3.2 \mathrm{GHz}$.

fluxes and to the ARCADE 2 frequencies. As summarized by Kellerman et al. (2008), a variety of VLA measurements indicate a trend toward a differential number count index of $\gamma=2.5$ in the faintest flux density bins, along with substantial scatter in the number density from different survey fields.

Our results, however, do not depend on measurements from a radio survey at any one frequency. Gervasi et al. (2008a) describe a more comprehensive analysis of the potential contribution of unresolved extragalactic radio sources by examining data from a wide variety of radio surveys, and fitting an empirical, twopopulation model to the survey data at each frequency. Their combined result for extragalactic radio source brightness versus frequency is described by a single power-law model, "Fit1," with amplitude $0.88 \mathrm{~K}$ at $0.61 \mathrm{GHz}$ and a power-law index of -2.707 . This model is also shown in Figure 1, indicating again that this contribution is insufficient for explaining the ARCADE 2 results. The brightness temperature values of this model, evaluated at the frequencies used in our analysis, appear in Table 1. Because the sample size and brightness limits of the radio surveys vary with frequency, the error in their estimate also varies with frequency. These frequencies are not identical to the frequencies measured by ARCADE 2. For Table 1, we adopt a fractional error estimate of $10 \%$ independent of frequency, which we believe is a conservative estimate of the error in the Gervasi et al. (2008a) modeling. These estimates for the contribution of extragalactic sources are consistent with the analysis described above.

It is also interesting to consider whether the existing surveys have missed significant flux from the sources. The low-frequency faint-source observations are primarily interferometric and have the possibility of overresolving the source and missing flux in extended low surface brightness emission. Henkel \& Partridge (2005) consider the evidence for this and conclude that $20 \%$ may be an upper limit to this effect for mJy flux levels at $8.5 \mathrm{GHz}$. Fomalont et al. (2006) suggest that at $1.4 \mathrm{GHz}$ only a few percent of sources are larger than $4 \operatorname{arcsec}$ and that other reports of a larger figure in other surveys 
are actually confusion of multiple disparate sources. Garrett et al. (2000) compare their $1.4 \mathrm{GHz}$ survey conducted using the Westerbork Synthesis Radio Telescope and its larger, 15 arcsec effective beam with previous VLA measurements of the same region with a similar noise level. Of a total of 85 sources in their survey, they find 22 not apparent in the previous VLA survey. Some of these 22 likely correspond to the combined flux of multiple sources that were resolved by the VLA measurements. At least two sources, however, appear to be relatively nearby discrete sources with emission from a large enough region to have been resolved out by the VLA measurements. We conclude from these studies that it seems unlikely that sufficient flux has been missed in surveys of known objects to explain our residual emission.

Another method to examine the possibility of extended low surface brightness emission in extragalactic sources is radio observations of the halos of nearby edge-on spirals. Irwin et al. $(1999,2000)$ report results of VLA surveys for radio emission from nearby edge-on spirals. These studies can elucidate the connection between the star formation processes that drive the far-IR background, and the supernova processes that drive radio emission, but do not provide evidence that large amounts of radio flux are missed in surveys of more distant sources.

\subsection{Connection with Far-IR Background}

The cosmic far-IR background has been detected at a level of approximately $10-20 \mathrm{nW} \mathrm{m}^{-2} \mathrm{sr}^{-1}$ with FIRAS and DIRBE (Puget et al. 1996; Fixsen et al. 1998; Hauser et al. 1998). We can use the universal radio to far-IR correlation in star-forming galaxies (Condon 1992) to estimate the expected extragalactic radio background that can be attributed to galaxies contributing to the cosmic far-IR background. Haarsma et al. (2000) and Dwek \& Barker (2002) specifically address this prospect. The conclusion of these studies is that the far-IR measurements are consistent with the existing surveys of radio galaxies described earlier. For example, the radio brightness temperature of $18 \mathrm{~K}$ at $178 \mathrm{MHz}$ predicted by Dwek \& Barker (2002) is within $1 \sigma$ of the radio galaxy contribution modeled by Gervasi et al. (2008a). This emission is insufficient to account for the excess detected by ARCADE 2.

One can ask if there is a way around this limit by considering departures from the far-IR radio correlation associated with faintness or redshift; the physical processes of far-IR emission from dust heated by star formation and radio emission driven by supernovae are related but differ in timescale (see, e.g., Murphy et al. 2008). Garn \& Alexander (2009) stack IR-selected galaxies and data from faint radio surveys and find that there is no evidence for a change in the far-IR to radio correlation with fainter galaxies. While some authors present the possibility that the far-IR correlation evolves with redshift (Vlahakis et al. 2007; Seymour et al. 2009), others see no evidence for this (Chapman et al. 2005; Frayer et al. 2006). In this paper, we follow Dwek $\&$ Barker (2002) and assume the measured correlation to hold in deriving our estimate for the contribution to the radio signal from star-forming galaxies.

\section{FIT OF MODEL SPECTRUM}

Here we fit a variety of model spectra to the combined data sets of ARCADE, lower frequency radio surveys, and the higher frequency results from FIRAS, after subtraction of an estimated point-source contribution. For subtraction of the point-source contribution, we have used the Gervasi et al. (2008a) "Fit1" model described in Section 3.1. For ARCADE and the lower frequency radio surveys, we use an estimate of the sky temperature after subtraction of a model of the emission from our own Galaxy, as described by Kogut et al. (2011). The associated errors are correlated between frequencies; we therefore use the noise covariance as outlined by Fixsen et al. (2011), which includes off-diagonal elements. For FIRAS data, we use the simplification of reducing the data to one point at $250 \mathrm{GHz}$ with $1 \mathrm{mK}$ error. The choice of $250 \mathrm{GHz}$ for the frequency of this data point (as opposed to say, $280 \mathrm{GHz}$ ) makes an insignificant difference in the values of the fit parameters described below.

For the sake of comparison, we have also investigated using a larger FIRAS data set of temperatures measured at 43 discrete frequencies and include the noise covariance among the data points. This covariance also includes the contribution from the covariance among the data points due to the common uncertainty in the temperature of the FIRAS external calibrator. The covariance matrix is described by Brodd et al. (1997). We have also included a nuisance parameter times a frequency model of Galactic dust as determined by FIRAS, which reduces the degrees of freedom in the result by one, as described by Fixsen et al. (1996).

In what follows, we will refer to this set as the "full FIRAS" data set, while the simplification of FIRAS to one point we will refer to as the "condensed FIRAS" data point. The comparison between these sets is only relevant for the chemical potential distortion fitting described below, as this distortion is the only one of those considered that has sufficient signal in the FIRAS frequency range for the "full FIRAS" data set to have an advantage over the simpler "condensed FIRAS" data set. Correspondingly, we will use the "condensed FIRAS" data set for all of the fitting procedures described below except in the case of chemical potential distortions, where we will compare the two.

The form of the fitting function is

$$
T(v)=T_{0}^{\mathrm{Ant}}(v)+A(v / 0.31 \mathrm{GHz})^{\beta}+\Delta T(v),
$$

where $T_{0}^{\mathrm{Ant}}(v)$ is the CMB baseline temperature (expressed in antenna temperature units), $A$ is the power-law amplitude at $0.31 \mathrm{GHz}, \beta$ is the power-law index, $v$ is the frequency, and $\Delta T(v)$ is a CMB spectral distortion (also expressed in antenna temperature units). The choice of $0.31 \mathrm{GHz}$ as the pivot frequency in Equation (3) helps minimize the covariance between the amplitude and index in the resulting fits; this choice is described in detail by Fixsen et al. (2011).

Because ARCADE 2 uses a blackbody calibrator of known physical temperature, the resulting sky measurements have units of thermodynamic temperature. It is therefore necessary to convert this data to units of antenna temperature before fitting using

$$
T_{\text {Ant }}=\frac{h v / k}{e^{h v / k T_{\text {Phys }}}-1},
$$

where $h$ is Planck's constant, $k$ is Boltzmann's constant, $T_{\text {Ant }}$ is the antenna temperature, and $T_{\mathrm{Phys}}$ is the physical temperature. We use a Levenberg-Marquardt nonlinear least-squares minimization for the fitting procedure (Marquardt 1963). The parameters for the fits described in this section are summarized in Table 2. 
Table 2

Spectral Fits to Combined ARCADE 2, FIRAS, and Radio Survey Data

\begin{tabular}{lcccc}
\hline \hline \multicolumn{1}{c}{ Parameter } & Power-law Fit & Power Law $+Y_{\mathrm{ff}}$ & $Y_{\mathrm{ff}}$ Only & Power Law $+\mu$ Distortion \\
\hline$T_{\mathrm{CMB}}{ }^{\mathrm{a}}$ & $2.725 \pm 0.001$ & $2.725 \pm 0.001$ & $2.725 \pm 0.001$ & $2.725 \pm 0.001$ \\
Power-law amplitude $^{\mathrm{b}}$ & $18.4 \pm 2.1$ & $14.6 \pm 8.1$ & $\ldots$ & $14.6 \pm 3.4$ \\
Power-law index & $-2.57 \pm 0.05$ & $-2.65 \pm 0.22$ & $\ldots$ & $-2.66 \pm 0.10$ \\
Free-free amplitude & $\ldots$ & $0.17 \pm 0.33$ & $0.49 \pm 0.07$ & $\ldots$ \\
$\mu$ amplitude & $\ldots$ & $\ldots$ & $\ldots$ & $(-4.4 \pm 2.7) \times 10^{-4}$ \\
Degrees of freedom & 11 & 10 & 46.4 & 10 \\
$\chi^{2}$ & 17.4 & 17.1 & 3.87 & 14.8 \\
Reduced $\chi^{2}$ & 1.58 & 1.71 & & 1.48 \\
\hline
\end{tabular}

Notes.

a The best-fit thermodynamic temperature of the CMB in $\mathrm{K}$.

b The fit amplitude of a power-law component in $\mathrm{K}$ (antenna temperature) at $0.31 \mathrm{GHz}$.

c The fit amplitude of $\Delta T_{\mathrm{ff}}$ in $\mathrm{K}$ (antenna temperature) at $1 \mathrm{GHz}$.

\subsection{Power Law Plus CMB}

Figure 1 shows that there is clear excess emission detected by ARCADE 2 in the $3 \mathrm{GHz}$ channels compared to what is expected from the CMB plus the contribution of extragalactic radio sources. As noted above, the fit is performed on the combined data sets after subtraction of an estimate of the point-source contribution. The unexplained residual emission is consistent with a power law with amplitude $18.4 \pm 2.1 \mathrm{~K}$ at $0.31 \mathrm{GHz}$, with a spectral index of $\beta=-2.57 \pm 0.05$.

We have also experimented with inflating the assumed errors for the removal of extragalactic discrete sources. As noted in Section 3.1, we have assumed a fractional error of $10 \%$, independent of frequency, for the discrete source contribution model. Inflating this error to $50 \%$ fractional error makes much less than a $1 \sigma$ change in the values of our power-law fit parameters above, and only a small $(\sim 10 \%)$ increase in the quoted errors in the fit parameters. This is because the error in the removal of the discrete sources is smaller than the other errors in the low-frequency measurements, as can be seen by inspection of Table 1.

\subsection{Free-Free Distortions}

Free-free distortions to the CMB spectrum can arise from energy released at lower redshifts (Bartlett \& Stebbins 1991; Gnedin \& Ostriker 1997; Oh 1999) and can be characterized by

$$
\Delta T_{\mathrm{ff}}(v)=T_{0} \frac{Y_{\mathrm{ff}}}{x^{2}}
$$

where $Y_{\mathrm{ff}}$ is the optical depth to free-free emission, $T_{0}$ is the undistorted $\mathrm{CMB}$ temperature, $x$ is the dimensionless frequency $h v / k T_{0}$, and $\Delta T$ is apparent temperature distortion.

A lower limit can be placed on the optical depth to free-free emission from late time effects of $Y_{\mathrm{ff}}>8 \times 10^{-8}$ (Haiman \& Loeb 1997). The current upper limit of $Y_{\mathrm{ff}}<1.9 \times 10^{-5}$ comes from combining data from FIRAS and previous ground-based CMB spectrum measurements (Bersanelli et al. 1994).

We have performed a four-component fit (Equation (3)) to the data to assess whether there is evidence to support a free-free spectral distortion to the $\mathrm{CMB}$, compared to the three-parameter fit described in the previous section. The four-fit components consist of a constant CMB temperature, a power-law amplitude, a power-law index, and a free-free amplitude. The fit parameters are presented in Table 2 . The addition of the free-free amplitude does not improve the reduced $\chi^{2}$ of the fit and is therefore not justified by the data.
We have also examined a two-parameter fit consisting of a constant CMB component and free-free distortion component; the parameters are shown in Table 2. This fit produces a significantly worse reduced $\chi^{2}$ and is therefore not consistent with the source of the unexplained emission.

The $2 \sigma$ limits on the free-free amplitude at $1 \mathrm{GHz}$ derived from our four-parameter fit are $-0.49 \mathrm{~K}<\Delta T_{\mathrm{ff}}<0.84 \mathrm{~K}$. This corresponds to an upper limit on the free-free optical depth of

$$
\left|Y_{\mathrm{ff}}\right|<1 \times 10^{-4} \text {. }
$$

This limit is less constraining than those reported by Bersanelli et al. (1994) and Gervasi et al. (2008b). This is the result of the additional degrees of freedom allowed by our four-parameter fit. As described above, the four-parameter fit is a better description of the data than the CMB plus free-free distortion fits performed by Bersanelli et al. (1994) and Gervasi et al. (2008b). We conclude that the tighter constraints offered by those studies are likely too optimistic.

It is interesting to consider how future measurements might improve the $Y_{\mathrm{ff}}$ limit. We have used the existing fits and asked how much tighter the limit becomes if an additional measurement of some fixed fractional accuracy is added to the data set. We have run this test as a function of frequency; the frequency range with the greatest effect is between 0.3 and $3.0 \mathrm{GHz}$, where a factor of several tighter constraints is potentially achievable. The results are shown in Figure 3, where we have plotted the size of the $2 \sigma$ errors on $Y_{\mathrm{ff}}$ as a function of the frequency of the additional measurement. We have assumed that the measurement point falls precisely on the existing fit. Better fractional error results in tighter constraints on $Y_{\mathrm{ff}}$. We have also considered the impact of repeating this test with additional measurements at 3,5, 8, 10, 30, and $90 \mathrm{GHz}$ with $0.002 \mathrm{~K}$ precision, as might be obtained with a future flight of ARCADE. Such a measurement would enhance the power of future lower frequency measurements to constrain $Y_{\mathrm{ff}}$ and would improve limits to free-free distortions better than any single new measurement at lower frequency.

\subsection{Chemical Potential $(\mu)$ Distortions}

Energy release early in the universe can distort the spectrum of the CMB. Energy injection at redshift $z \lesssim 10^{7}$ no longer results in a Planck spectrum, but instead forms a spectrum with a Bose-Einstein photon occupation number (Sunyaev \& Zeldovich 1970). An example of such an effect is the damping of primordial density perturbations before the epoch of recombination (Hu et al. 1994). 


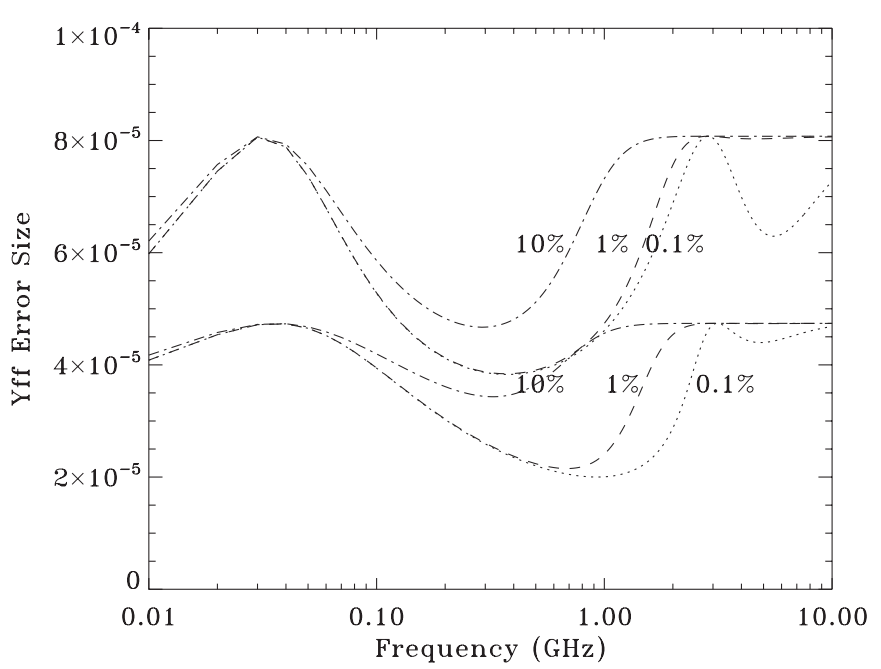

Figure 3. Improvement of limit on $Y_{\mathrm{ff}}$ obtainable with future measurements. Shown are the sizes of the $2 \sigma$ errors on $Y_{\mathrm{ff}}$ (upper set of three curves) that would result from an additional measurement at a frequency shown on the $x$-axis, with a precision of $0.1 \%$ (dotted curve), $1 \%$ (dashed curve), or $10 \%$ (dot-dashed curve). The lower set of three curves repeats this test under the assumption that there are additional measurements at $3,5,8,10,30$, and $90 \mathrm{GHz}$ with $0.002 \mathrm{~K}$ precision, as would result from a future flight of ARCADE 2. The additional measurement is assumed to be centered on the value of the existing fit.

A series of papers (Danese \& De Zotti 1980; Burigana et al. 1991a, 1991b, 1995) has investigated in detail the shape of the resulting spectral distortions after inclusion of free-free processes which act at the low-frequency end. The shape of the distortion depends on the range of redshift over which such energy injection takes place. We have used their analytic description of such distortions to the CMB to provide a functional form to fit to our combined data set. The occupation number is given by a Bose-Einstein like distribution with a frequency-dependent chemical potential:

$$
\eta_{\mathrm{BE}}=\frac{1}{e^{\left[x / \phi_{\mathrm{BE}}-\mu(x)\right]}-1},
$$

where

$$
\mu(x)=\mu_{0} \exp \left[-x_{c}\left(z_{1}\right) /\left(x / \phi_{\mathrm{BE}}\right)\right],
$$

$\mu_{0}$ is the chemical potential, $x \equiv h v / k T$ is the dimensionless frequency, and $\phi_{\mathrm{BE}}$ is the ratio of electron to radiation temperature with

$$
\phi_{\mathrm{BE}} \simeq\left(1-1.1 \mu_{0}\right)^{-1 / 4}
$$

The characteristic dimensionless frequency, $x_{c}\left(z_{1}\right)$, is given by an analytical approximation in Burigana et al. (1991a) and depends on the Hubble parameter, the baryon density, the present CMB temperature, and the number of relativistic species, $N_{\nu}$. For $N_{v}=3, T_{0}=2.725 \mathrm{~K}$, and $\Omega_{b} h^{2}=0.0227$ (Hinshaw et al. 2009), we find

$$
x_{c}\left(z_{1}\right) \simeq 5.7 \times 10^{-3},
$$

which corresponds to a frequency of approximately $0.33 \mathrm{GHz}$.

The above discussion neglects the contribution of additional photons at low energy through free-free emission. This effect can be included through the following modification of the occupation number (Danese \& De Zotti 1980):

$$
\eta=\frac{1-\left[1-\eta_{\mathrm{BE}}\left(e^{x / \phi_{\mathrm{BE}}}-1\right)\right] e^{-y_{\mathrm{abs}}(x)}}{\left[e^{x / \phi_{\mathrm{BE}}}-1\right]}
$$

Here, $y_{\mathrm{abs}}(x)$ is the optical depth to free-free absorption and depends on baryon density and other cosmological parameters. An analytic approximation is given in Burigana et al. (1991a).

We can compare the size of the corrections relative to a constant $\mu_{0}=9 \times 10^{-5}$ distortion, assuming the cosmological parameters described above. The correction to include a frequency-dependent chemical potential decreases the distortion amplitude by approximately $3 \mathrm{mK}$ at $1 \mathrm{GHz}$. The correction to include the contribution of additional photons at low energy through free-free emission decreases the distortion amplitude by $7 \mathrm{mK}$ at $1 \mathrm{GHz}$ relative to a constant $\mu$ distortion. The corrections described above become more important at frequencies below $1 \mathrm{GHz}$. We have included these corrections in our fits.

For fitting the data, the distorted spectrum is related to the occupation number by

$$
T_{\text {eff }}(v)=(h v / k) / \ln \left(1+\eta^{-1}\right) .
$$

The shape of the distortion is shown in Figure 4.

In addition to the $\mu$ distortion, we have included a power-law amplitude and index to the fit parameters. For this fit, we have used the "condensed FIRAS" data set. The addition of the $\mu$ distortion as a free parameter does not substantially improve the reduced $\chi^{2}$. The $2 \sigma$ upper limit on $\mu$ is

$$
\mu<6 \times 10^{-4} \text {. }
$$

This limit is not an improvement on the previous limit of $9 \times 10^{-5}$ set using FIRAS (Fixsen et al. 1996). The combination of the ARCADE 2 data and the "full FIRAS" data set (with proper inclusion of the FIRAS error covariance, including absolute calibration uncertainty, and a nuisance parameter times the Galactic dust model) does not result in a substantial improvement in the previous FIRAS limit.

We note that sensitivity to a chemical potential distortion from the data in Table 1 is dominated by the ARCADE data and the FIRAS data point, due to the large uncertainties in the lower frequency radio surveys. Over the range 3-90 GHz, the chemical potential distortion resembles a power law with a negative amplitude, making it degenerate with the power-law fit for the radio background. There is not enough curvature between 3 and $90 \mathrm{GHz}$ to precisely distinguish a chemical potential distortion from a power law, thus leading our less precise limit on $\mu$ from the low-frequency data alone.

\section{DISCUSSION}

We have presented evidence for isotropic radio emission detected by ARCADE 2 beyond what can be explained by our model of Galactic emission and the unresolved emission from the known population of discrete sources. The excess emission is consistent with a power law, with an index of -2.57 , which is significantly flatter than what might be expected from a population of faint, steep spectrum (index $\sim-2.7$ ) radio sources. We have also examined and placed limits on two classes of spectral distortions to the CMB. Such distortions are not supported by the data and cannot explain the excess emission, as is illustrated graphically in Figure 4.

It is possible to imagine that an unknown population of discrete sources exists below the flux limit of existing surveys. Such a population existing as a simple extension of the source counts of star-forming galaxies would be inconsistent with the two-population model of radio sources explored by Gervasi et al. (2008a). Such a population with a source count index 

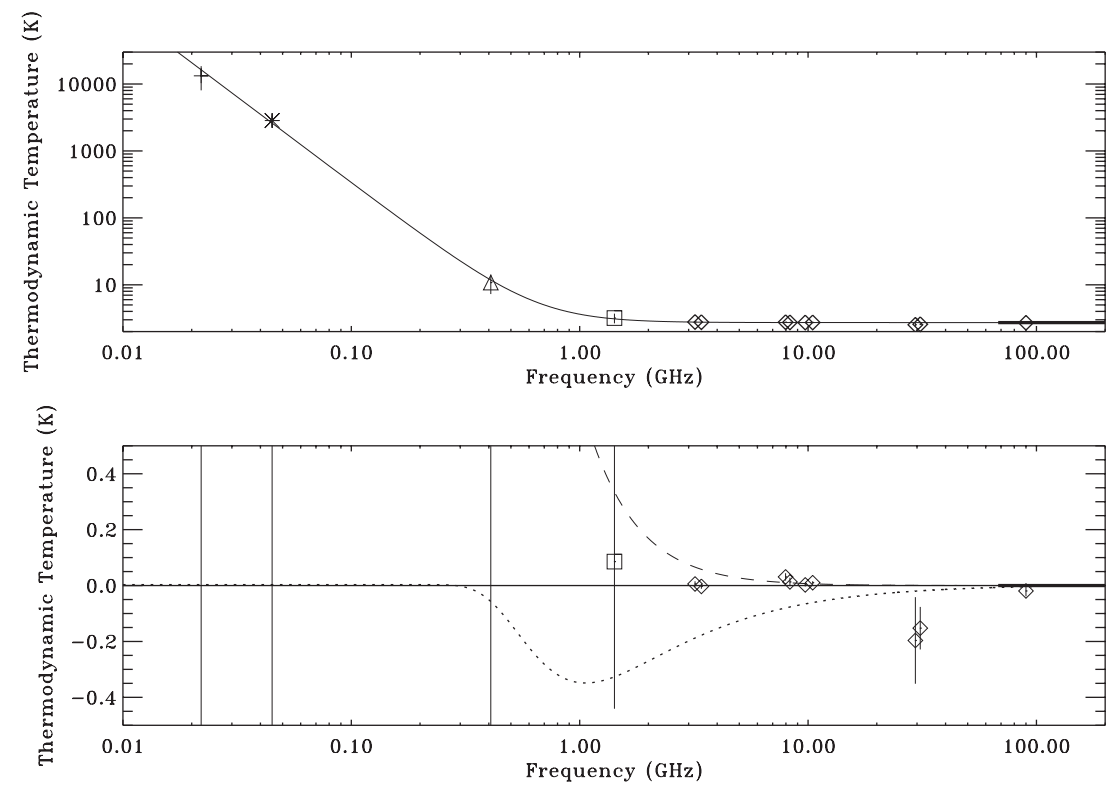

Figure 4. Fit of ARCADE 2 data, FIRAS data, and data from low-frequency radio surveys. The upper plot shows (solid line) a fit with three components: a frequencyindependent CMB contribution, a power-law amplitude, and a power-law index. The lower plot shows the fit residuals. The dotted line shows the expected shape of a $\mu$ distortion. The amplitude of the plotted distortion is 50 times the upper limit determined from FIRAS. The dashed line shows the shape of a $Y_{\mathrm{ff}}$ distortion with an amplitude equal to the $2 \sigma$ upper limit. The addition of either a $\mu$ distortion or a $Y_{\mathrm{ff}}$ distortion as a free parameter is not supported by the data. Data points are from Roger et al. (1999, cross), Maeda et al. (1999, asterisk), Haslam et al. (1981, triangle), Reich \& Reich (1986, square), ARCADE 2 (diamonds), and FIRAS (heavy line), corrected for Galactic emission and an estimate of extragalactic radio sources, as shown in Table 1.

close to $\gamma=2.5$ would have to extend to very low flux limits to explain our measurements and would require evading the radio to far-IR correlation noted earlier. It is interesting to consider to what extremes one would need to take the number counts in this analysis to account for the measured $62 \mathrm{mK}$ excess at $3.20 \mathrm{GHz}$. Using an index of $\gamma=2.5$ and extrapolating the number counts at $8.4 \mathrm{GHz}$ from Fomalont et al. (2002), we find that we would need to extend the lower flux limit to $0.3 \mathrm{nJy}$, which would result in a source density of $\sim 8 \times 10^{5} \mathrm{arcmin}^{-2}$. As a toy model, we can also consider a population of sources distributed with a delta function in flux a factor of 10 fainter than the $8.4 \mathrm{GHz}$ survey limit of Fomalont et al. (2002). At a flux of $0.75 \mu \mathrm{Jy}$, it would take over 1100 such sources per square arcmin to produce the unexplained emission we see at $3.20 \mathrm{GHz}$, assuming a frequency index of -2.57 . This source density is only modestly greater than the surface density of objects revealed in the faintest optical surveys, e.g., the Hubble Ultra Deep Field (Beckwith et al. 2006).

It is important to consider modeling and instrumental sources of error. Underestimating the level of Galactic emission is a potential contaminant. As described by Kogut et al. (2011), the expected contribution of the area around the North Galactic Pole is $\sim 10 \mathrm{~K}$ at $0.31 \mathrm{GHz}$, with relatively tight errors. The isotropic residual, therefore, is incompatible with emission tied to known Galactic structure, but could reside in a halo or other isotropic component. Our modeled Galactic emission and the residual emission presented here have similar spectral indices. We are therefore unable to exclude Galactic modeling errors as an explanation for the residual emission on the basis of spectral index.

Correcting for instrumental systematic errors in measurements such as ARCADE 2 is always a primary concern. We emphasize that we detect residual emission at $3 \mathrm{GHz}$ with the ARCADE 2 data, but the result is also independently detected by a combination of low-frequency data and FIRAS. The unexplained emission is detected at several $\sigma$ with any two of the three data subsets: (1) FIRAS and low-frequency radio data, (2)
ARCADE 2 and low-frequency radio data, and (3) ARCADE 2 and FIRAS. The result is therefore robust to problems in any one measurement.

We conclude that the residual signature is due either to a diffuse extragalactic background of emission from discrete radio sources with properties somewhat different than the faint end of the distribution of known sources or to unmodeled residual emission from our own Galaxy. Although we believe the former to be more likely, we cannot exclude the latter explanation. Further observations with sufficient sensitivity to distinguish between these on the basis of spectral index may clarify which explanation is correct.

It is a pleasure to thank the staff of the Columbia Scientific Balloon Facility for their support of ARCADE 2. We thank the undergraduate students whose work helped make ARCADE 2 possible: Adam Bushmaker, Jane Cornett, Sarah Fixsen, Luke Lowe, and Alexander Rischard. ARCADE 2 was supported by the National Aeronautics and Space Administration suborbital program. T.V. acknowledges support from CNPq grants 466184/00-0, 305219-2004-9, and 303637/2007-2-FA, and the technical support from Luiz Reitano. C.A.W. acknowledges support from CNPq grant 307433/2004-8-FA. The research described in this paper was performed in part at the Jet Propulsion Laboratory, California Institute of Technology, under a contract with the National Aeronautics and Space Administration.

\section{REFERENCES}

Bartlett, J. G., \& Stebbins, A. 1991, ApJ, 317, 8

Beckwith, S. V., et al. 2006, AJ, 132, 1729

Bersanelli, M., Bensadoun, M., de Amici, G., Levin, S., Limon, M., Smoot, G. F., \& Vinje, W. 1994, ApJ, 424, 517

Bersanelli, M., Smoot, G. F., Bensadoun, M., de Amici, G., Limon, M., \& Levin, S. 1995, Astrophys. Lett. Commun., 32, 7

Brodd, S., Fixsen, D. J., Jensen, K. A., Mather, J. C., \& Shafer, R. A. 1997, COBE Far Infrared Absolute Spectrophotometer (FIRAS) Explanatory Supplement, COBE Ref. Pub. No. 97-C (Greenbelt, MD: NASA-GSFC)

Burigana, C., Danese, L., \& De Zotti, G. 1991a, A\&A, 246, 49 
Burigana, C., Danese, L., \& De Zotti, G. 1991b, ApJ, 379, 1

Burigana, C., De Zotti, G., \& Danese, L. 1995, A\&A, 303, 323

Chapman, S. C., Blain, A. W., Smail, I., \& Ivison, R. J. 2005, ApJ, 622, 772

Condon, J. J. 1992, ARA\&A, 30, 575

Danese, L., \& De Zotti, G. 1980, A\&A, 84, 364

Dwek, E., \& Barker, M. K. 2002, ApJ, 575, 7

Fixsen, D. J., Cheng, E. S., Gales, M., Mather, J. C., Shafer, R. A., \& Wright, E. L. 1996, ApJ, 473, 576

Fixsen, D. J., Dwek, E., Mather, J. C., Bennett, C. L., \& Shafer, R. A. 1998, ApJ, 508,123

Fixsen, D. J., Kogut, A., Levin, S., Limon, M., Lubin, P., Mirel, P., Seiffert, M., \& Wollack, E. 2004, ApJ, 612, 86

Fixsen, D. J., \& Mather, J. C. 2002, ApJ, 581, 817

Fixsen, D. J., et al. 2011, ApJ, 734, 5

Fomalont, E. B., Kellermann, K. I., Cowie, L. L., Capak, P., Barger, A. J., Partridge, R. B., Windhorst, R. A., \& Richards, E. A. 2006, ApJS, 167, 103

Fomalont, E. B., Kellermann, K. I., Partridge, R. B., Windhorst, R. A., \& Richards, E. A. 2002, AJ, 123, 2402

Frayer, D. T., et al. 2006, AJ, 131, 250

Garn, T., \& Alexander, P. 2009, MNRAS, 394, 105

Garrett, M. A., de Bruyn, A. G., Giroletti, M., Baan, W. A., \& Schilizzi, R. T. 2000, A\&A, 361, L41

Gervasi, M., Tartari, A., Zannoni, M., Boella, G., \& Sironi, G. 2008a, ApJ, 682, 223

Gervasi, M., et al. 2008b, ApJ, 688, 24

Gnedin, N. Y., \& Ostriker, J. P. 1997, ApJ, 486, 581

Gush, H. P., Halpern, M., \& Wishnow, E. H. 1990, Phys. Rev. Lett., 65, 537

Haarsma, D. B., Partridge, R. B., Windhorst, R. A., \& Richards, E. A. 2000, ApJ, 544,641

Haiman, Z., \& Loeb, A. 1997, ApJ, 483, 21

Haslam, C. G. T., Klein, U., Salter, C. J., Stoffel, H., Wilson, W. E., Cleary, M. N., Cooke, D. J., \& Thomasson, P. 1981, A\&A, 100, 209

Hauser, M. G., et al. 1998, ApJ, 508, 25

Henkel, B., \& Partridge, R. B. 2005, ApJ, 635, 950

Hinshaw, G., et al. 2009, ApJS, 180, 225

Hu, W., Scott, D., \& Silk, J. 1994, ApJ, 430, L5

Irwin, J. A., English, J., \& Sorathia, B. 1999, AJ, 117, 2102
Irwin, J. A., Saikia, D. J., \& English, J. 2000, AJ, 119, 1592

Johnson, D., \& Wilkinson, D. 1987, ApJ, 313, L1

Kellerman, K. I., Fomalont, E. B., Mainieri, V., Padovani, P., Rosati, P., Shaver, P., Tozzi, P., \& Miller, N. 2008, ApJS, 179, 71

Kogut, A., et al. 2011, ApJ, 734, 4

Levin, S., Bensadoun, M., Bersanelli, M., de Amici, G., Kogut, A., Limon, M. \& Smoot, G. 1992, ApJ, 396, 3

Maeda, K., Alvarez, H., Aparici, J., May, J., \& Reich, P. 1999, A\&AS, 140, 145

Marquardt, D. W. 1963, J. Soc. Ind. Appl. Math., 11, 431

Mather, J. C., Fixsen, D. J., Shafer, R. A., Mosier, C., \& Wilkinson, D. T. 1999, ApJ, 512, 511

Mather, J. C., et al. 1990, ApJ, 354, L37

Murphy, E. J., Helou, G., Kenney, J. D. P., Armus, L., \& Braun, R. 2008, ApJ, 678,828

Oh, S. P. 1999, ApJ, 527, 16

Owen, F. N., \& Morrison, G. E. 2008, AJ, 136, 1889

Puget, J.-L., Abergel, A., Bernard, J.-P., Boulanger, F., Burton, W.B., Désert, F.-X., \& Hartmann, D. 1996, A\&A, 308, L5

Raghunathan, A., \& Subrahmnayan, R. 2000, J. Astrophys. Astron., 20, 1

Reich, P., \& Reich, W. 1986, A\&AS, 63, 205

Richards, E. A. 2000, ApJ, 533, 611

Roger, R. S., Costain, C. H., Landecker, T. L., \& Swerdlyk, C. M. 1999, A\&AS, 137, 7

Rogers, A. E., \& Bowman, J. D. 2008, AJ, 136, 641

Seymour, N., Huynh, M., Dwelly, T., Symeonidis, M., Hopkins, A., McHardy, I. M., Page, M., \& Rieke, G. 2009, MNRAS, 398, 1573

Singal, J., et al. 2006, ApJ, 653, 835

Singal, J., et al. 2011, ApJ, 730, 138

Staggs, S., Jarosik, N. C., Meyer, S. S., \& Wilkinson, D. T. 1996a, ApJ, 473, L1

Staggs, S., Jarosik, N. C., Wilkinson, D. T., \& Wollack, E. J. 1996b, ApJ, 458, 407

Sunyaev, R. A., \& Zeldovich, Ya. B. 1970, Ap\&SS, 7, 20

Vlahakis, C., Eales, S., \& Dunne, L. 2007, MNRAS, 379, 1042

Windhorst, R. A., et al. 1993, ApJ, 405, 498

Zannoni, M., Tartari, A., Gervasi, M., De Lucia, A., Passerini, A., Cavaliere, F., Boella, G., \& Sironi, G. 2008, ApJ, 688, 12 